MODELING, IDENTIFICATION AND CONTROL, 1987, VOL. 8, NO. 1, 1-10

doi:10.4173/mic.1987.1.1

\title{
Biomass estimation in aquaculture facilities
}

\section{A. LQVIK $\dagger$}

Keywords: Acoustics, fish, length distribution, aquaculture.

The paper discusses the problem of size determination of fish without any handling requirements. Specifically acoustical remote sensing based on the resonance of the swimbladder is shown theoretically and experimentally.

\section{Introduction}

Aquaculture is a growing industry in Norway and many other countries. The species to be produced vary from country to country and so do the production plant and methods. In facilities where fish are produced a number of similar instrumentation needs exist for

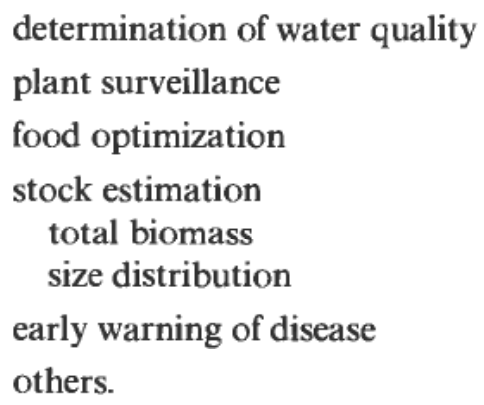

The justification for improved instrumentation is given by a number of factors

increased distance to the base facility

use of automatic feeding systems

market demand for a continuous supply

increased competition

Possible solutions to the determination of biomass and fish size distribution are discussed. The instrumentation is intended for fish with swimbladders like salmon, trout or cod, and results obtained are taken from measurements on different species, both free swimming in a shoal or in cages. In the following a discussion of the measuring problem is given followed by a discussion on possible measuring methods, acoustical aspects and some preliminary results.

\section{Measuring problem}

The aim of the instrumentation is to give an estimate of the total biomass in a cage and the size distribution of the fish within a cage or a vessel. The problem is

Received 15 January 1987.

This paper was presented at the IFAC Symposium on Automation and Dataprocessing in Aquaculture, Trondheim, Norway, 18-20 August 1986.

This paper is reprinted with the permission of IFAC.

† Bird Laboratories A/S, Wergelandsv. 3, 7500 Stjørdal, Norway. 
divided into two parts

a coarse estimate for surveillance

a precise assessment when the fish are sorted in size groups

The first instrumentation will give the total biomass in a cage and the size distribution without handling the fish. The second will count the fish and determine their size when passing through a measuring zone while being sorted in size groups. This measurement will give the most accurate result of the two, but it involves some kind of handling. In the following an outline of solutions to the first problem will be given. The results from this remote sensing system will give the total biomass, and the size distribution from which growth rate, changes in biomass and the need for size sorting may be estimated.

\section{Measuring method}

Remote sensing of a biomass is a well known problem in fisheries research, where today acoustic methods are in use. The echo integration technique using calibrated echo sounders gives estimates of the total biomass. In order to find the size distribution of the surveyed fish population the only acoustical method in use today is based on the analyses of echoes from single fish. Thus the method is limited to spatially thin schools. As the acoustic frequency used is normally in the order of $20-100 \mathrm{kHz}$ the back scattering is directive and special statistical and acoustical methods have to be applied in order to find the representative back scattering strength.

In most cages used in Norwegian aquaculture facilities the fish density is very high and the traditional methods are not applicable. Thus methods measuring on the ensemble have to be used. The method to be described is shown schematically in
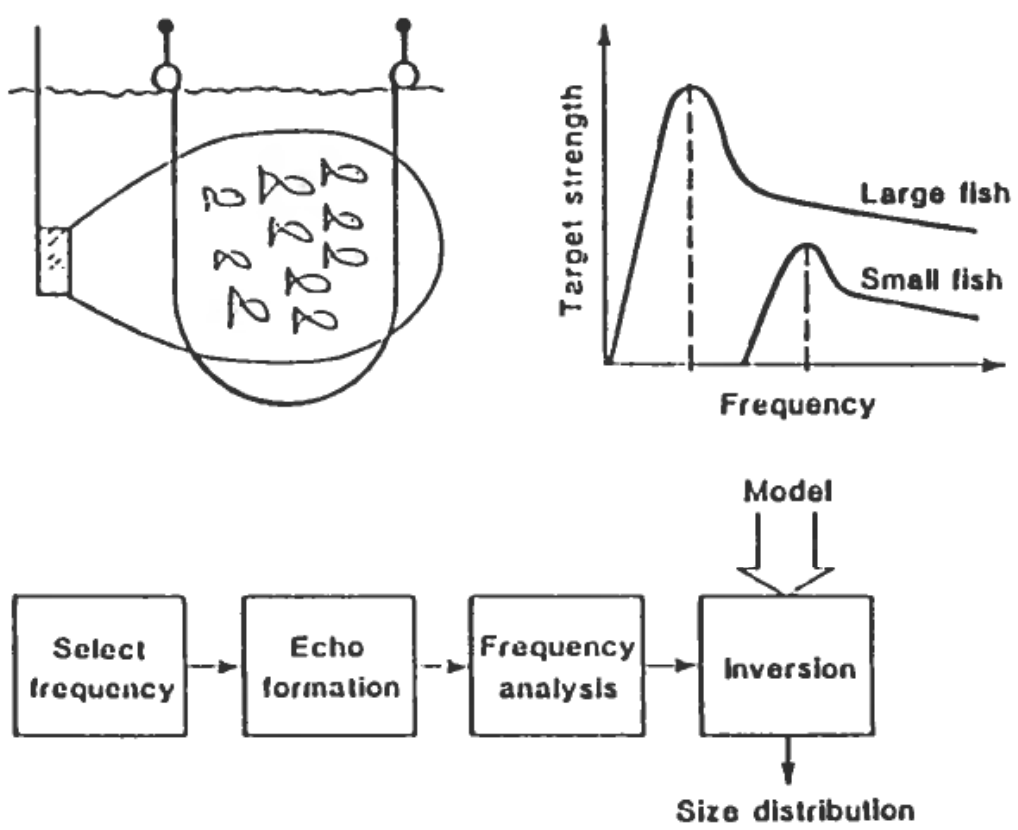

Figure 1. Basic processes of fish length estimation by resonance echo sounding. 
Fig. 1. The method may also be extended for use in any enclosed area like a large basin or a fjord with a narrow inlet.

The fishes are ensonified with low frequency sound, $0 \cdot 1-10 \mathrm{kHz}$, i.e. in the resonance frequency region of the swimbladders of the fishes, the backscattering is almost omnidirectional, and as demonstrated in Fig. 1, a large fish will have a high backscattering strength and a low resonance frequency. In a closed volume either backscattering or absorption may be the significant variable. The received echo or attenuated signal is frequency analysed, and compared with the acoustic model of the process to give an estimate of the fish size distribution.

\section{Acoustic properties of the swimbladder in the resonance region}

On the basis of previous investigation (Andreeva, 1964; McCartney and Stubbs, 1971; Holliday, 1972) together with Løvik and Hovem (1979) it may be concluded that the properties of the swimbladder of fish may be adequately described by modeling the bladder as a gas filled sphere.

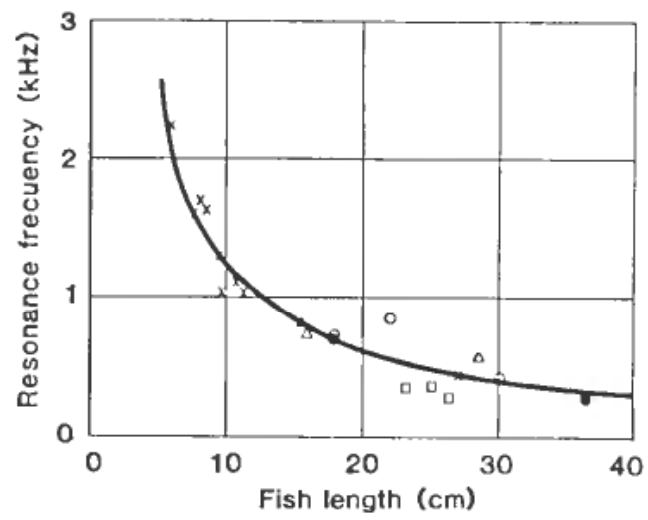

Figure 2. Resonance frequency versus fish length.

Figure 2 summarizes the relationship between the resonance frequency and the fish length of various species. The obtained resonance frequencies are normalized to a reference depth $(1 \mathrm{~m})$. The results indicate an inverse relationship between resonance frequency, $f_{0}$, and fish length, $L$

$$
f_{0}=K_{1} / L
$$

where $K_{1}$ is found to be between 110 and 120 when $f_{0}$ is in hertz and $L$ in metres. The spread of the data points due to different species is not found to be consistent. The reasons for the lower resonance frequencies for cod and the higher ones for herring may be that the resonance frequencies of gas cavities having equal total volume will increase as the cavity becomes more elongated (Weston, 1967).

The inverse linear relationship, eqn. (1), implies that the effective swimbladder radius is proportional to the fish length and that the volume is proportional to the length cubed. This is certainly not always fulfilled due for instance to seasonal variation in fat content, thus adjustments of eqn. (1) are expected. The adaptation to depth has been studied by transferring the fish from one depth to which it has been adapted to another depth where it was left for a new state of adaptation.

Typical results are displayed for the resonance frequency and $Q$-value of a saithe, $77 \mathrm{~mm}$ long, in Figs. 3 and 4. The resonance frequency in Fig. 3 is found to increase 


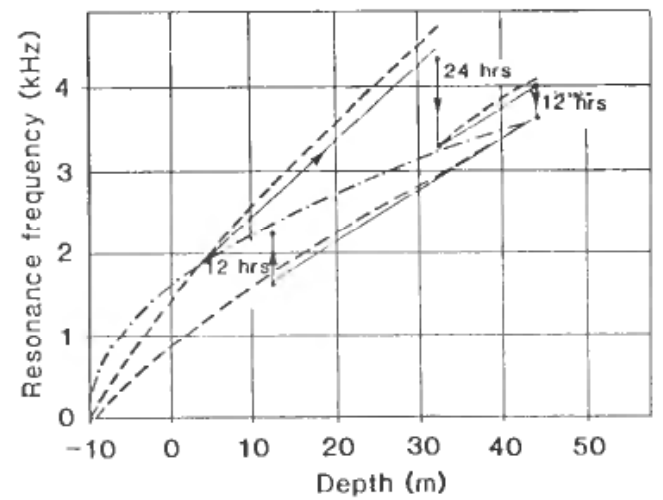

Figure 3. Resonance frequency versus depth. --- the law of constant gas mass, $-\cdot-\cdots \cdot-$ the law of constant gas volume, and —_measured values.

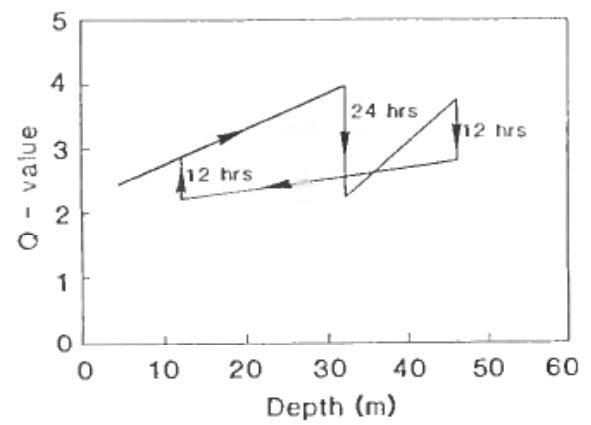

Figure 4. $Q$-value versus depth.

with the square root of the ambient pressure for an adapted fish. The transfer is done while the mass of the gas is constant, giving a higher or lower resonance frequency than the adapted one depending on the direction of transfer. The two mechanisms of adaptation may be described as follows:

1. the law of constant gas volume:

$$
f_{0}(d)=f_{0}\left(d_{0}\right)\left(\frac{10+d}{10+d_{0}}\right)^{1 / 2}
$$

where $d$ is depth and $f_{0}\left(d_{0}\right)$ is the resonance frequency at a depth $d_{0}$.

2. the law of constant gas mass:

$$
f_{0}(d)=f_{0}\left(d_{0}\right)\left(\frac{10+d}{10+d_{0}}\right)^{5 / 6}
$$

Adaptation is thus a process of adjusting the gas content to maintain a constant volume of the swimbladder, and then neutral buoyancy.

If the fish normally lives at a given depth say in a cage and only swims up in order to catch some food our results from free swimming fish seem to confirm the hypothesis that the fish is adapted to some average depth and migrates around this depth without significant secretion of absorption of gas.

This is illustrated in Fig. 5. When the fish is at a greater depth, pressure $\boldsymbol{P}_{\boldsymbol{m} 2}$, than the adaptation depth, pressure $P_{0}$, the swimbladder volume is reduced and the 


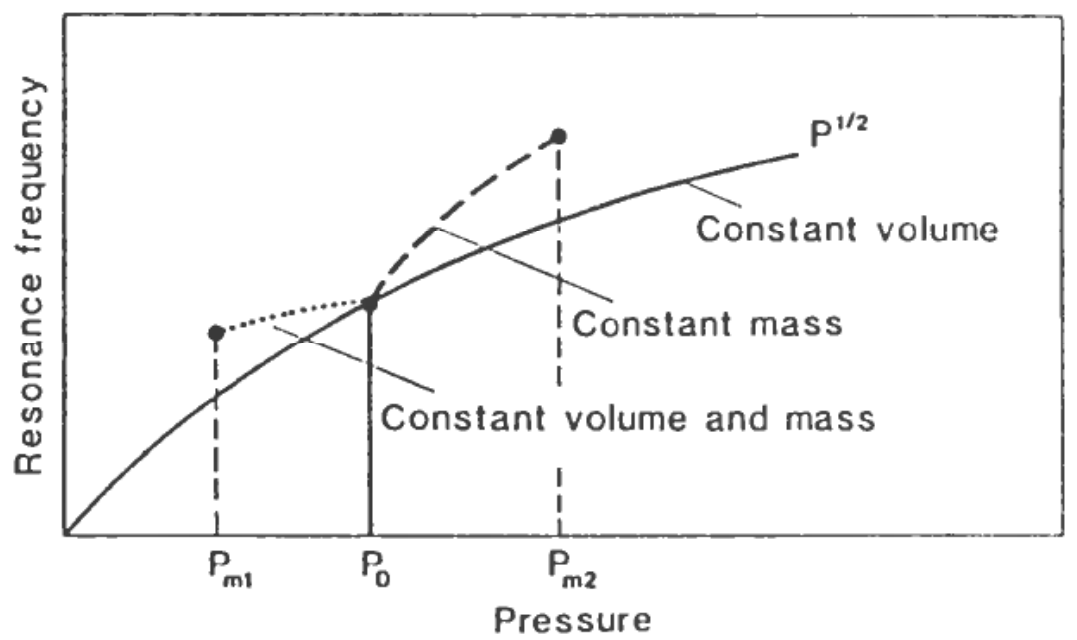

Figure 5. Adaptation mechanisms.

fish must increase the effort to swim or may rest for ease on the bottom. At shallower depths, pressure $P_{m 1}$, the volume of the bladder is controlled by muscle activities and possibly some absorption. The adaptation also seems to be dependent on species or type of swimbladder. The $Q$-value, Fig. 4 , is fairly constant or shows a slight trend to increase with depth for an adapted fish. Again the unadapted values are higher or lower than the adapted ones depending on the direction of transfer.

Measurements of the gas volume from the fishes under study yield a relationship between fish length and an equivalent swimbladder radius. From this it is possible to find the theoretical target strength of a fish and compare it with observed values, as shown in Fig. 6.

The data is obtained from echo measurements on saithe and the correspondence between the theoretical and the measured values is felt to be good. The model seems to describe the scattering at low frequencies and in the vicinity of the resonance frequency. At frequencies much higher than the resonance region the model is probably less accurate.

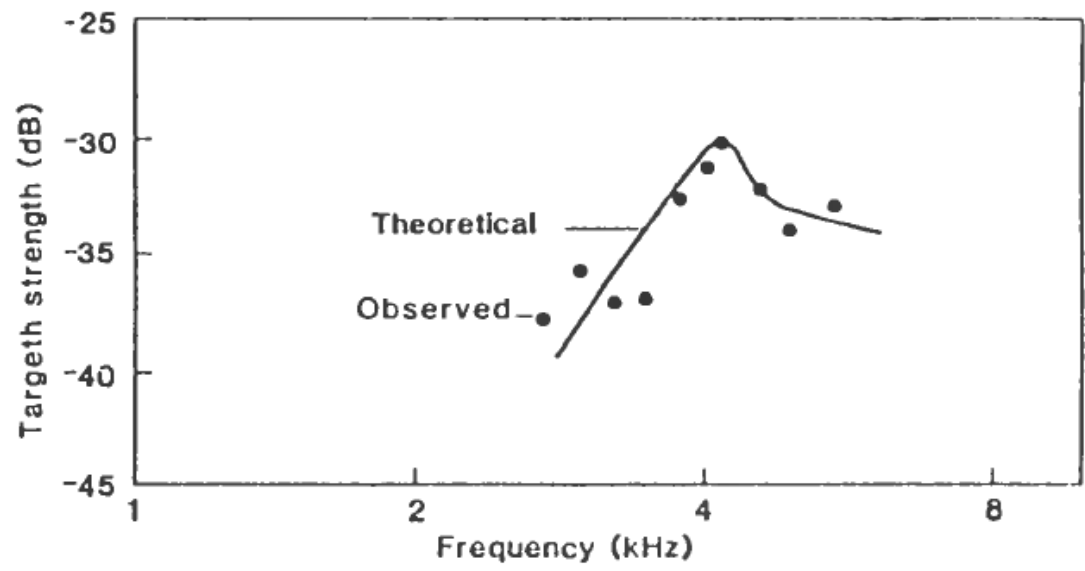

Figure 6. Target strength as a function of frequency. 


\section{The inversion model}

The main objective is to estimate fish length from measurements at several frequencies covering the expected range of resonance frequencies of fish present.

Assuming measurement are done at $m$ discrete frequencies of the backscattering cross-section or a related quantity, an echo matrix $S$ having elements $S_{i}$ are formed

$$
S=\left(\begin{array}{lllll}
S_{1} & S_{2} & S_{3} & \ldots & S_{m}
\end{array}\right)^{T}
$$

At the frequency $f_{i}$ the total predicted backscattering cross section is expressed as $C_{i}$ :

$$
C_{i}=\sum_{j=1}^{n} \sigma_{i j} N_{j}
$$

$\sigma_{i j}$ is an element of the scattering matrix $\sigma$, i.e. the backscattering cross section at $f_{i}$ for a fish in the length interval $j$ of totally $n$ intervals. $N_{j}$ is an element of the number matrix $N$, i.e. the number of organisms within each length interval $j$. Equation (5) is only valid in the case of negligible multiple scattering, no shadowing and incoherent scattering.

The inversion is based on a least squares approximation, trying to minimize the square of the difference between the measured echoes $\boldsymbol{S}$ and the theoretical echoes C.

$$
\min (\boldsymbol{S}-\boldsymbol{C})^{2}
$$

The solution of the problem is

$$
N=\left(\sigma^{T} \sigma\right)^{-1} \sigma^{T} S
$$

Thus knowing the backscattering matrix and having the measured echoes, the number of scatterers could be found.

The solution given by eqn. (7) has however a severe limitation in the fact that the number matrix $N$ may be negative, leading to a restatement of eqn. (6)

$$
\min (S-C)^{2}
$$

when

$$
N \geqslant 0
$$

If it is assumed that the backscattering is given mainly by scattering from the swimbladder, the backscattering cross section, $\sigma_{b}$, as a function of frequency, $f_{i}$, is given by:

$$
\sigma_{b}\left(f_{i}\right)=\frac{a_{j}^{2}}{\left(\left(f_{0} / f_{i}\right)^{2}-1\right)^{2}+\sigma^{2}}
$$

where $a_{j}$ is the equivalent radius of the swimbladder, $f_{0}$ is the resonance frequency and $\sigma$ is the damping constant. If the measurements are based on attenuation, the extinction cross section $\sigma_{e}$ should be used

$$
\sigma_{c}(f)=\frac{4 \pi a\left(\sigma / \sigma_{R}\right)}{\left(\left(f_{0} / f\right)^{2}-1\right)^{2}+\sigma^{2}}
$$

where $\sigma_{R}$ is the attenuation factor at resonance. Both cross sections show a dominant peak at resonance. An illustration of the extinction cross-section is shown in Fig. 7. 


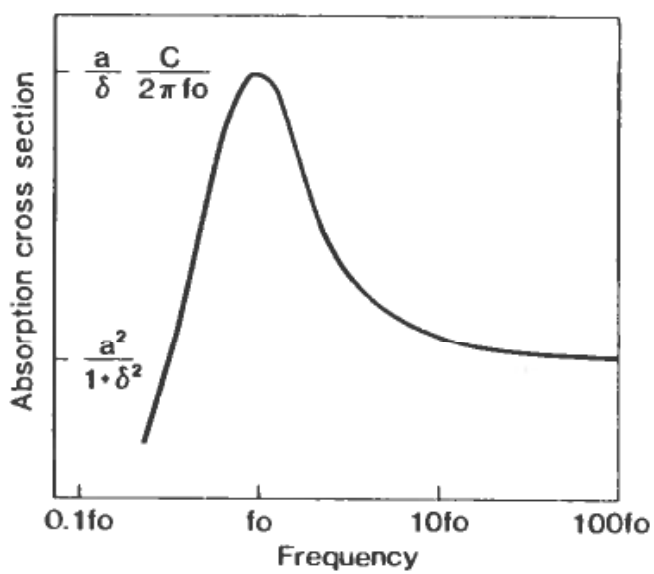

Figure 7. Extinction cross-section.

\section{Results}

Some results from measurements on free swimming fish and on salmon in a cage are given. The two situations involve, for the free swimming fish, the use of the returned echo and for the caged fish the attenuation through the cage.

The instrumentation in both cases is a bi-static multi-frequency sonar system as shown in Fig. 8. The recorder synchronizes both the frequency generator and the filterset to a pre-calibrated recording paper, the gating system controls the pulse length, the repetition rate and the depth or range window where the received signals are measured. The measured signals are presented as a function of frequency on the recorder, and recorded on tape. Normally the sonar is not used in a continuous frequency scanning mode, but at a predetermined set of frequencies one at a time. At each frequency typically 50-100 transmissions are used to obtain an average response.

A typical set of field measurements consist of the following

equipment calibration

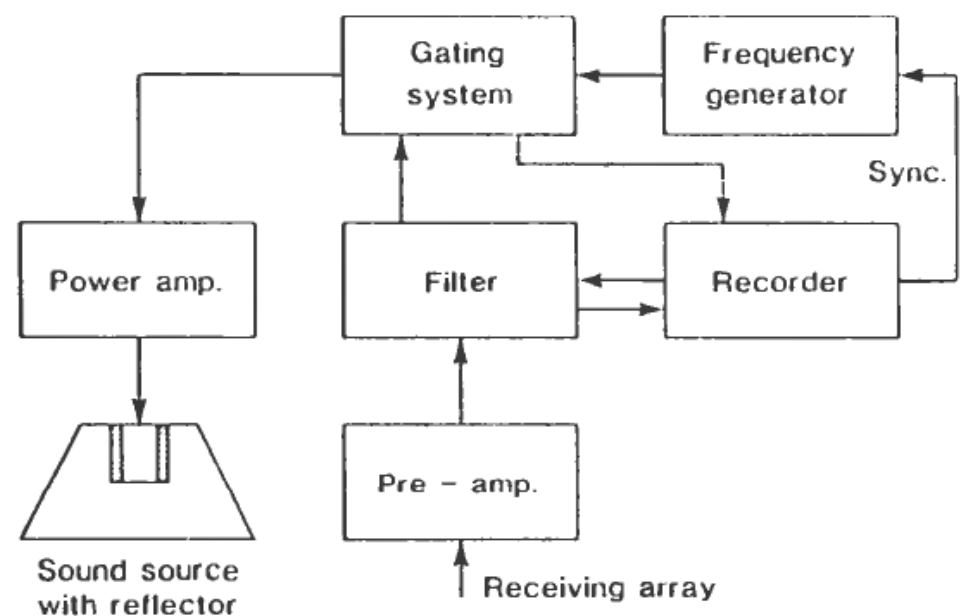

Figure 8. Block diagram of the sonar system. 


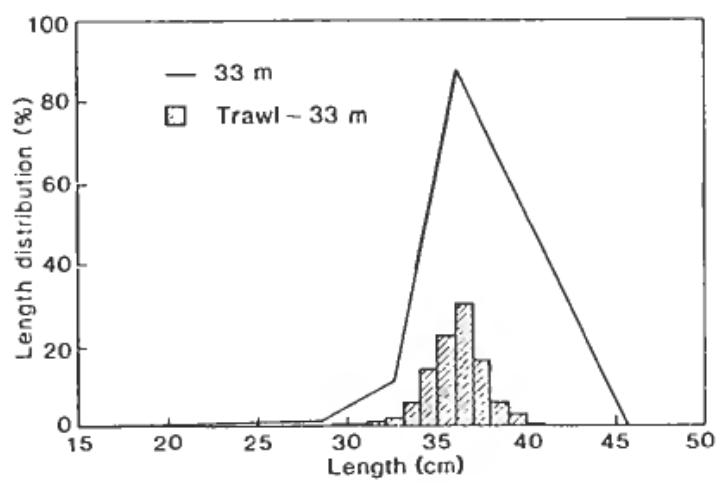

Figure 9. Acoustically determined length distribution and length distribution from trawl catch.

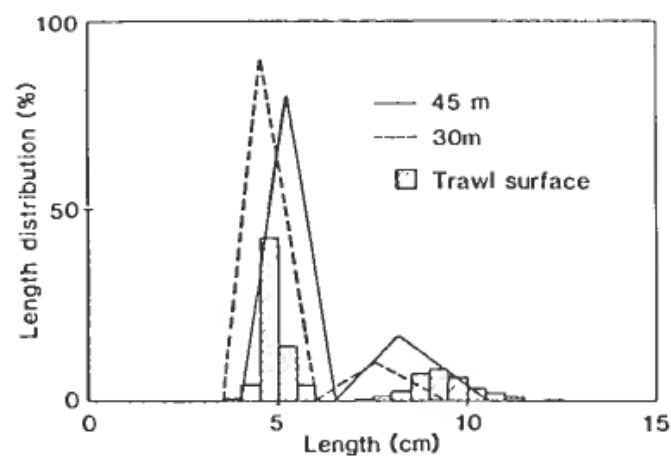

Figure 10. Acoustically determined length distribution and length distribution from trawl catch.

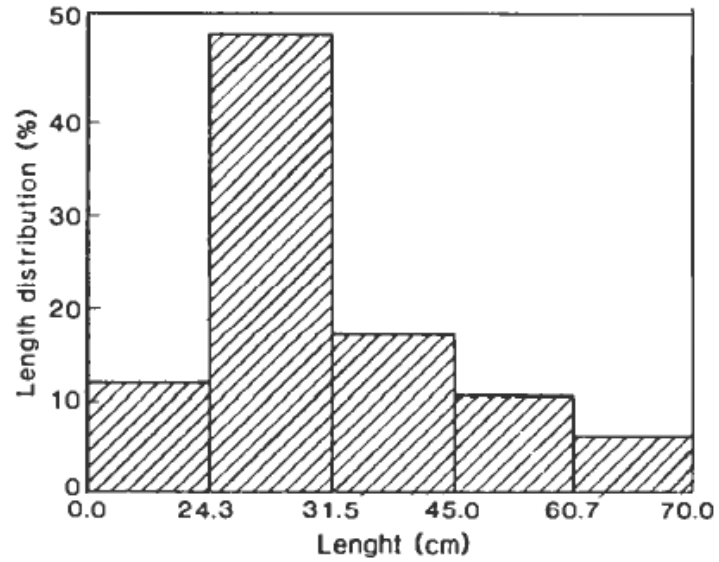

Figure 11. Acoustically determined length distribution for salmon. 
measurement, echo/attenuation of fish

biological sampling either by trawling or use of landing net, followed by manual classification

The received acoustical signals are processed on shore, so far. The inversion algorithms used are given by eqns. (8), (9) and (10) with the proper calibration and propagation corrections applied.

Figures 9 and 10 show results from free swimming shoals. The correspondence between the trawl data and the acoustically determined data is fairly good for both distributions shown. The method also seems to work for small as well as larger fish.

Figure 11 shows the results from attenuation measurement on fairly large salmon in a $12 \mathrm{~m}$ diameter net cage. The fish were not ready for slaughter and a limited number of fish was taken up and measured. The mean fish length was found to be around $35 \mathrm{~cm}$, well in agreement with the acoustically determined length histogram.

\section{Concluding remarks}

The work described has shown that the use of acoustical methods may give fair estimates of the fish size distribution. The method based on the swimbladder resonance yields good results for various species and lengths of fish.

It will give the fish farmer a significant knowledge of his stock to be able to judge growth rate, loss or damage, and the need for size grouping of the population.

In a separate but closely linked project the problem of counting while sorting is being solved, thus eventually a complete and unique biomass estimation and size sorting package will be available to the fish farmer.

\section{REFERENCES}

ANDREEVA, I. B. (1964). Scattering of sound by air bladders of fish in deep sound-scattering ocean layers. Sov. Phy.-Acoust., 10, 17-20.

Holliday, D. V. (1972). Resonance structure in echoes from schooled pelagic fish. J. Acoust. Soc. Am., 51, 1322-1332.

LøviK, A., and HOVEM, J. (1979). An investigation of swimbladder resonance in fishes. $J$. Acoust. Soc. Am., 66, 850-854.

MCCARTNEY, B. S., and StuBbS, A. R. (1971). Measurements of target strength of fish in donal aspect, including swimbladder resonance. $J$. Sound, Vib., 15, 397-420.

WESTON, D. E. (1967). Sound propagation in the presence of bladder fish, in Underwater acoustics, ed. by V. M. Albess (Plenum Press, New York). 